\title{
RESONANT PHONONS IN ADSORBED SLABS
}

\author{
L. Dobrzynski, A. Akjouj, B. Sylla and B. Djafari-Rouhani
}

Equipe Internationale de Dynamique des Interfaces

Laboratoire de Dynamique et Structure des Matériaux Moléculaires U.R.A. au C.N.R.S. $n^{\circ}$ 801, U.F.R. de Physique, Université de Lille I 59655 Villeneuve d'Ascq Cedex, France

(Received May 21, 1991)

Resonant phonons, sometimes also called leaky waves, are phonons associated with a crystal defect, a surface, an adsorbed layer and whose frequencies fall inside the bulk crystal band. Such resonant phonons were studied experimentally and theoretically before for clean surfaces and for adsorbed monolayers. We present here a study of resonant phonons associated with the adsorption of a slab of $L$ monolayers on a substrate. With the help of a simple atomic model, we obtained a closed form expression giving the variation of the transverse phonon density of states associated with the adsorption of the slab. An application which qualitatively simulates the adsorption of $L$ monolayers of $\mathrm{Ge}$ on $\mathrm{Si}$ shows the existence of well-defined resonant phonons within the bulk acoustic band of the substrate.

PACS numbers: $68.60 .-p$

In the last two decades there have been numerous measurements of localized phonons on clean surfaces and on surfaces with adsorbed monolayers using inelastic electron [1] and $\mathrm{He}$ [2] scattering. The long wavelength limit of these excitations is studied also by using interdigital transducer techniques [3] and Brillouin scattering [4] and is used for many applications [3]. Raman investigations of the vibration of thin slabs adsorbed on a substrate just begins $[5,6]$.

Although resonant phonons were detected on clean surfaces and on surfaces with one adsorbed monolayer, no such experimental nor theoretical study exists yet to our knowledge for resonant phonons associated with several adsorbed monolayers.

We present here a simple lattice dynamical model which enables us to obtain a closed form expression for the variation of the phonon density of states associated with the adsorption of a finite number $L$ of monolayers of a given adsorbate on a substrate. A numerical application of this result which simulates the case of a Ge slab adsorbed on Si shows the existence of peaks within the acoustic bulk band of $\mathrm{Si}$, which are the signature of the resonant phonons.

The atomic model used here was fully described before [7] in connection with a study of localized phonons within a slab sandwiched between two other 
semi-infinite crystals. Let us just stress here its main characteristics and define its parameters.

We use a simple cubic lattice of atoms of mass $m_{1}$ to describe the semi-infinite substrate, whose (001) atomic planes are labelled by the integer $n_{3}$ such that $n_{3} \leq 0$. The adsorbed slab has the same structure and lattice parameter $a_{0}$ as the substrate and has $L(001)$ planes of atoms of mass $m_{2}$ such that $1 \leq n_{3} \leq L$. The phonon model takes into account only the first nearest neighbour atom interactions $\beta_{1}$ for the substrate, $\beta_{2}$ for the slab and $\beta_{I}$ between substrate and adsorbate atoms. As in this model there is no interaction between the three different components $u_{\alpha}(n)(\alpha=1,2,3)$ of the displacement of the atom situated at lattice site $x(n)=a_{0}\left(n_{1} \widehat{x}_{1}+n_{2} \widehat{x}_{2}+n_{3} \widehat{x}_{3}\right)$, the phonons given by this model are three times degenerate. Despite its simplicity this model well describes the transverse phonons having a polarization perpendicular to the sagittal plane which contains the propagation vector $k_{\|}=k_{1} \widehat{x}_{1}+k_{2} \widehat{x}_{2}$, parallel to the (001) surface and the vector $\widehat{x}_{3}$ perpendicular to the surface.

From the expressions of the response function $g\left(n_{3}, n_{3} ; k_{\|}, \omega^{2}\right)$, where $\omega$ stands for the frequency of these transverse phonos, given in Ref. [7], we calculate the variation $\Delta n\left(k_{\|}, \omega^{2}\right)$ of the phonon density of states between the crystal with the $L$ adsorbed layers described before and a crystal where the $L$ layers would be replaced by $L$ layers of the substrate. In both systems we have therefore the same number of atoms and vibrational modes. We choose these two situations to compare in view of light scattering experimental investigations, in which the probe deeply penetrates into the crystals with and without the adsorbed layer. We calculated therefore

$$
\Delta n\left(k_{\|}, \omega^{2}\right)=-\frac{1}{\pi} \operatorname{Im} \sum_{n_{3}}\left[g\left(n_{3}, n_{3} ; k_{\|}, \omega^{2}\right)-g_{\mathbf{s}}\left(n_{3}, n_{3} ; k_{\|}, \omega^{2}\right)\right],
$$

where $g_{\mathrm{s}}\left(n_{3}, n_{3} ; k_{\|}, \omega^{2}\right)$ is the response function of the semi-infinite substrate having the same number of atomic planes as the sample with the $L$ adsorbed monolayers. A closed form expression for $\Delta n\left(k_{\|}, \omega^{2}\right)$ was obtained as a function of the following quantities:

$$
\begin{aligned}
& t_{i}\left(k_{\|} \omega^{2}\right)=\left\{\begin{array}{lc}
\xi-\left(\xi_{i}^{2}-1\right)^{1 / 2}, & \xi_{i}>1, \\
\xi_{i}+i\left(1-\xi_{i}^{2}\right)^{1 / 2} & -1<\xi_{i}<1, \\
\xi_{i}+\left(\xi_{i}^{2}-1\right)^{1 / 2} & \xi_{i}<-1
\end{array}\right. \\
& \xi_{i}=3-\cos k_{1} a_{0}-\cos k_{2} a_{0}-\frac{m_{i}}{2 \beta_{i}}\left(\omega^{2}+i \varepsilon\right), \quad i=1 \text { or } 2 \text {, } \\
& W\left(k_{\|}, \omega^{2}\right)=\beta_{1} \beta_{2}\left(t_{1}-1\right)\left(t_{2}-1\right)\left(1-t_{2}^{2 L}\right) \\
& -\beta_{\mathrm{I}}\left[\beta_{2} t_{1}\left(t_{2}-1\right)\left(1-t_{2}^{2 L}\right)+\beta_{1}\left(t_{1}-1\right)\left(t_{2}+t_{2}^{2 L}\right)\right],
\end{aligned}
$$

where $\varepsilon$ is an infinitesimal small number.

The above definitions enable us to give now the expression of

$$
\Delta n\left(k_{\|}, \omega^{2}\right)=-\frac{1}{\pi} \operatorname{Im}\left\{\frac{-m_{1} t_{1}}{\beta_{1}\left(t_{1}^{2}-1\right)}\left[L+\frac{\beta_{1} \beta_{2}}{W} \frac{\dot{t}_{1}}{t_{1}-1}\left(t_{2}-1\right)\left(1-t_{2}^{2 L}\right)\right]\right.
$$




$$
\begin{aligned}
& +\frac{m_{2} t_{2}}{\beta_{2}\left(t_{2}^{2}-1\right)}\left[-\frac{t_{2}}{t_{2}+1}\left(2 \beta_{1} \beta_{2}\left(t_{2}-1\right)-\beta_{\mathrm{I}}\left[2 \beta_{2} t_{1}+\beta_{1}\left(t_{1}-1\right)\right]\right) \frac{1-t_{2}^{2 L}}{W}\right. \\
& +\frac{L}{W}\left(\beta_{1} \beta_{2}\left(t_{1}-1\right)\left(t_{2}-1\right)\left(1+t_{2}^{2 L}\right)-\beta_{1}\left[\beta_{2} t_{1}\left(t_{2}-1\right)\left(1+t_{2}^{2 L}\right)\right.\right. \\
& \left.\left.\left.\left.+\beta_{1}\left(t_{1}-1\right)\left(t_{2}-t_{2}^{2 L}\right)\right]\right)\right]\right\} .
\end{aligned}
$$

In the application to the adsorption of a Ge slab on a $\mathrm{Si}$ substrate, we assumed $\beta_{2} / \beta_{1}=\beta_{\mathrm{r}} / \beta_{1}=1$ and $m_{2} / m_{1}=2.58$ and represented the variation $\Delta n\left(k_{\|}, \omega\right)$ of the phonon density of states per unit of $\omega$, which is related to $\Delta n\left(k_{\|}, \omega^{2}\right)$ by the following expression:

$$
\Delta n\left(k_{\|}, \omega\right)=2 \omega \Delta n\left(k_{\|}, \omega^{2}\right) \text {. }
$$

But let us first show on Fig. 1, for three adsorbed monolayers, the connection within the two-dimensional Brillouin zone between the localized and resonant modes. The figure presents $\Omega=\omega / \omega_{\max }$, where $\omega_{\max }$ is the maximum frequency

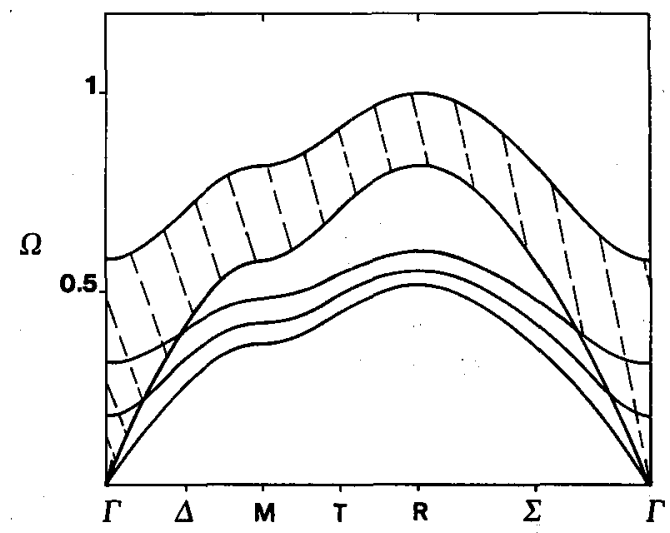

Fig. 1. Localized and resonant transverse phonon dispersion curves within the two-dimensional Brillouin zone due to three adsorbed monolayers of $\mathrm{Ge}$ on $\mathrm{Si}$ such that $\Omega=\omega / \omega_{\max }, \beta_{2} / \beta_{1}=1$ and $m_{2} / m_{1}=2.58$.

of the substrate phonons, as a function of $a_{0} k_{\|}$. Starting from the center $F$ of the two-dimensional Brillouin zone, we go to the point $M(\pi, 0)$ along the axis $\Delta\left(a_{0} k_{1}, 0\right)$, then to the point $R(\pi, \pi)$ along the axis $T\left(\pi, a_{0} k_{2}\right)$ and finally back to $\Gamma$ through the axis $\Sigma\left(a_{0} k_{1}, a_{0} k_{2}\right)$. The bulk phonon band of the substrate lies in the shaded region. Below it there are three branches of phonons associated with the three layers of the adsorbed $\mathrm{Ge}$. The lowest of these three branches corresponds to a localized mode in the whole two-dimensional Brillouin zone. The two others become resonant modes when they penetrate within the substrate bulk band. In order to study if these resonances remain well-defined features and could be detected experimentally, we calculated the corresponding variation of the density of states as defined above. Figures 2 and 3 present these results respectively for 


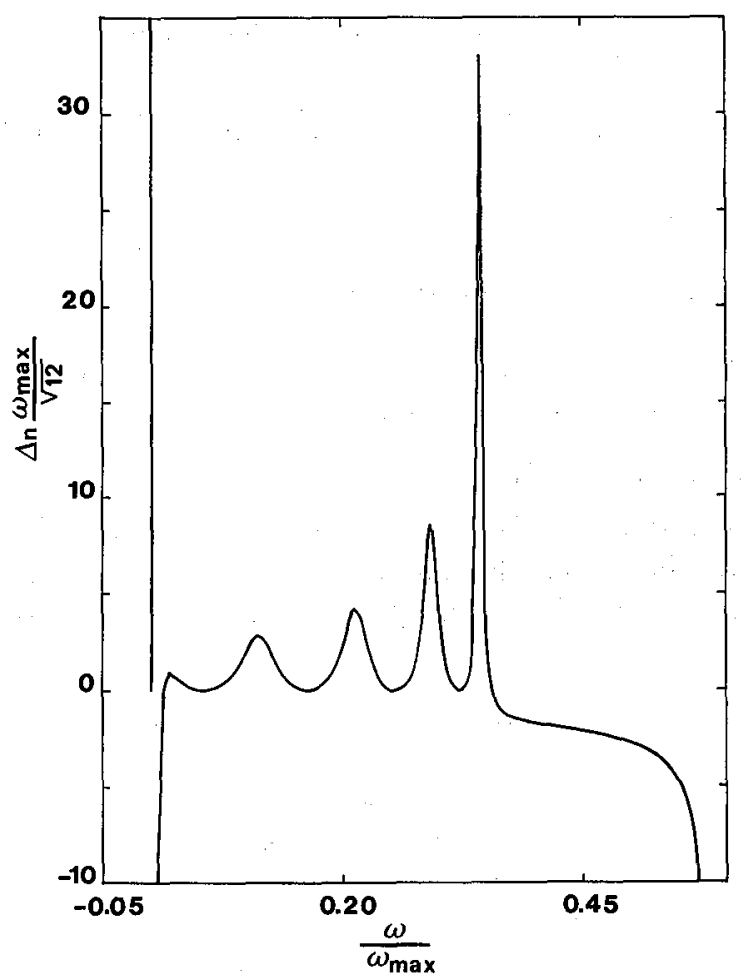

Fig. 2. Variation of the transverse phonon density of states $\Delta n\left(k_{\|}, \omega / \omega_{\max }\right)$ multiplied by $\omega_{\max } / \sqrt{12}$ for five adsorbed monolayers of $\mathrm{Ge}$ on $\mathrm{Si}$, for $k_{\|} a_{0}=\pi / 100$.

$L=5$ and $L=10$. In order to have quantities without dimension we represented $\Delta n\left(k_{\|}, \omega / \omega_{\max }\right) \omega_{\max } / \sqrt{12}$ as a function of $\omega / \omega_{\max }$ for $k_{\|} a_{0}=\pi / 100$. Starting from low frequencies; we see first a delta peak at the frequency of the localized mode and then respectively 5 resonances (for $L=5$ ) and 9 resonances (for $L=10$ ). The first resonance for $L=5$ is not the continuation of a localized mode as discussed before in Fig. 1. This resonance was found to exist in the region in Fig. 1 lying between the bottom of the bulk band and the lowest resonance associated with a localized mode. As the above defined region becomes smaller when $L$ increases and exists only for small values of $k_{\|} L a_{0}$, this resonance exists only for small values of $k_{\|} L a_{0}$, and is already only a weak feature in Fig. 3 for $k_{\|} a_{0} L=\pi / 10$. The other resonances are well-defined features and exists for any value of $L$ in the region where the bulk bands of $\mathrm{Ge}$ and $\mathrm{Si}$ intersects. Let us notice that we obtained $L-1$ such resonances for all values of $L$ and that their sharpness increases with $L$. We observe also that the amplitude of the peaks increases with $\omega / \omega_{\max }$.

The experimental observation of these resonances will have to be discussed in the future in connection witl the resolution of each experimental technique. Let us finally say that we also studied [8] these resonances within the frame of 


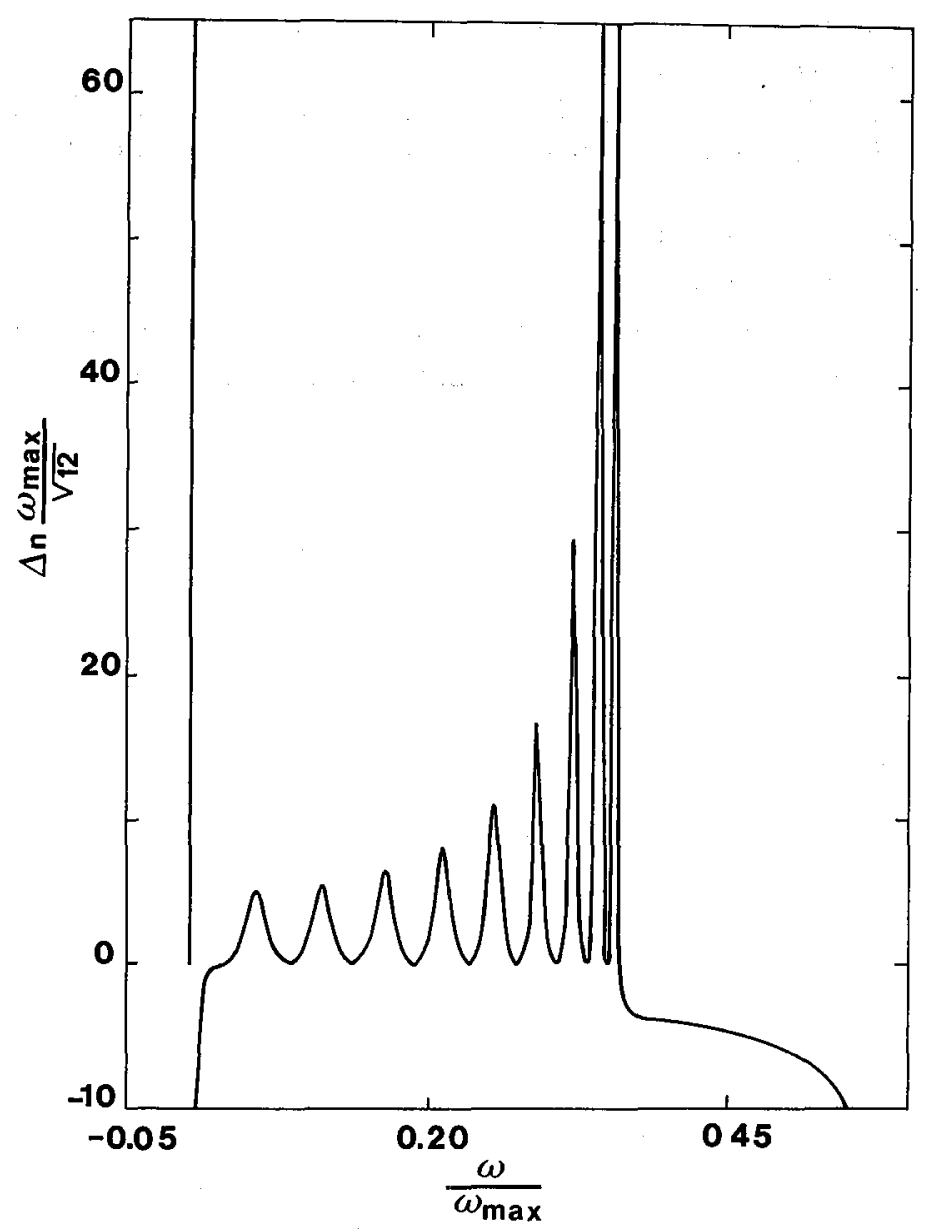

Fig. 3. Same as Fig. 2, but for ten adsorbed monolayers of $\mathrm{Ge}$ on $\mathrm{Si}$. Note that the amplitude of the highest frequency peak was found to be 234 and falls outside the frame of this figure.

elasticity theory. This study confirms the present results and the corresponding variation of the density of states is in agreement with the results given here for the lowest frequency peaks. As there is no top for the bulk phonon band in the elasticity model, such a model cannot give the increase in the amplitude of the peaks and their separation for the highest frequencies.

We hope that these preliminary results will stimulate further theoretical and experimental studies of resonances in thin adsorbed slabs. 


\section{References}

[1] See for example: H. Ibach, J. Vac. Sci. Technol. A 5(4), 419 (1987).

[2] See for example: J.P. Toennies, J. Vac. Sci. Technol. A 5(4), 440 (1987).

[3] See for example: B.A. Auld, in Acoustic Fields and Waves in Solids, Vol. 2, Wiley-Interscience, New York 1973.

[4] See for example: V. Bortolani, F. Nizzoli, G. Santoro, J. Phys. Collog. C5-151 (1984); F. Nizzoli, J.R. Sandercock, in: Dynamical Properties of Solids, Eds. G.K. Horton, A.A. Maradudin, Vol. 6, p. 281 (1990).

[5] D. Lockwood, private communication.

[6] N. Ovsyuk, private communication.

[7] A. Akjouj, B. Sylla, P. Zielinski, L. Dobrzynski, J. Phys. C 20, 6137 (1987).

[8] A. Akjouj, B. Sylla, B. Djafari-Rouhani, L. Dobrzynski, to be published. 